\title{
Instability of Propiconazole Resistance and Fitness in Monilinia fructicola
}

\author{
K. D. Cox, P. K. Bryson, and G. Schnabel
}

Department of Entomology, Soils, and Plant Sciences, 120 Long Hall, Clemson University, Clemson, SC 29634.

Current address of the first author: Department of Plant Pathology, Barton Laboratory, New York State Agricultural Experiment Station, Cornell University, Geneva 14456.

Accepted for publication 13 October 2006.

\begin{abstract}
Cox, K. D., Bryson, P. K., and Schnabel, G. 2007. Instability of propiconazole resistance and fitness in Monilinia fructicola. Phytopathology $97: 448-453$.

The fitness and the dynamics of demethylation inhibitor fungicide (DMI) sensitivity in isolates of Monilinia fructicola sensitive (no growth at $0.3 \mathrm{mg} /$ liter propiconazole) and resistant ( $\geq 50 \%$ relative growth at 0.3 $\mathrm{mg} /$ liter propiconazole) to propiconazole were investigated. Overall, there was no considerable compromise in the fitness of resistant isolates compared to sensitive isolates of $M$. fructicola at the time of collection. Resistant and sensitive isolates differed in their sensitivity to propiconazole $(P$ $<0.001)$ and incubation period $(P=0.044)$, but not in latent period, growth rate, spore production, and spore germination frequency $(P>$

0.05). Consecutive transferring on potato dextrose agar had an impact on conidia production, conidial germination, and growth rate $(P<0.0001)$. Consecutive transferring also had an impact on propiconazole sensitivity in resistant isolates. In the resistant isolates, sensitivity to propiconazole increased $\left(R^{2}=0.960, P=0.0034\right)$ within the first eight transfers. Similarly, sensitivity to propiconazole increased by $273 \%$ over the course of 34 months in cold storage in propiconazole-resistant isolates. Our results show that propiconazole resistance is unstable in vitro and that standard subculturing and cold storage procedures impact propiconazole sensitivity of resistant isolates. The instability of propiconazole resistance in M. fructicola may have important implications for disease management in that a reversion to propiconazole sensitivity could potentially occur in the absence of DMI fungicide pressure in the field.
\end{abstract}

Monilinia species cause many diseases of fruit crops worldwide including blossom blight and fruit rot of stone fruits (caused by M. laxa, M. fructigena, and M. fructicola), cranberry cottonball (caused by $M$. oxycocci), mummy berry disease of blueberry (caused by $M$. vaccinii-corymbosi), and leaf and fruit blight of Rhododendron (Monilinia jezoensis), among others $(1,28)$. In the southeastern United States Monilinia species cause two diseases which greatly impact the fruit industry: brown rot of peach [M. fructicola (G. Wint.) Honey] and mummy berry disease of blueberry (M. vaccinii-corymbosi).

Currently, application of fungicides is the most effective means of managing diseases caused by Monilinia spp. Since the development of widespread benzimidazole resistance in the early 1980s (31), demethylation inhibitor fungicides (DMIs) have become one of the most effective and widely-used fungicides for Monilinia management $(24,30)$. The triazoles propiconazole, tebuconazole, and fenbuconazole have become industry standards for chemical management of brown rot of peach and of mummy berry disease of blueberry in the Southeastern United States (8). Despite a history of success for over 15 years using DMIs for brown rot control in this region, there have been recent reports of control failures and reduced sensitivity in $M$. fructicola isolates from Georgia $(2,24)$. There have been no reports of M. fructicola control failure using DMIs in South Carolina, but reduced sensitivity to propiconazole has been reported in some isolates from South Carolina (30). In recent years, the Quinone outside inhibitor fungicides (QoI), a class of fungal respiration inhibitor fungicides, have been registered and are now recommen-

Corresponding author: G. Schnabel; E-mail address: schnab@clemson.edu

doi:10.1094/PHYTO-97-4-0448

(c) 2007 The American Phytopathological Society ded for use in rotation with DMIs to control brown rot in the region (8).

The development and stability of fungicide resistance in a population of fungi is in part dependent on the parasitic, vegetative, and reproductive fitness of the resistant members. The stability of fungicide resistance and selection for fungicide resistance in a population will be lessened if resistant members have reduced fitness such as growth, sporulation, or ability to cause disease. For example, benzimidazole resistance has been stable for several decades and benzimidazole-resistant and -sensitive isolates of several fungal species are reported to be equally fit and equally parasitic $(9,18,20,27)$. Similarly, dichloranresistant and -sensitive isolates of Rhizopus stolonifer do not differ in pathogenicity (29) and dichloran resistance is considered to be fairly stable. In contrast, reduced fitness of dicarboximideresistant isolates has been reported for several ascomycetes, including Penicillium expansum (23), Botrytis cinerea (5), and M. fructicola (22), despite the fact that dicarboximide resistance is well established.

Several studies have used fitness compromise and sensitivity decline following consecutive propagation or cold storage as a means of gauging the stability of DMI resistance in fungi (10-12). Currently, little is known regarding fitness compromise and resistance stability in propiconazole-resistant $M$. fructicola isolates in the Southeastern United States. Since DMIs are still important for management of diseases caused by Monilinia spp. in the region, fitness and sensitivity decline following consecutive transfer and cold storage in propiconazole-resistant isolates merits investigation.

The purpose of this study was to investigate the dynamics of propiconazole sensitivity and fitness of $M$. fructicola isolates representative of populations in the southeastern United States. The specific objectives of the study were (i) to assess the fitness 
of propiconazole-resistant and -sensitive isolates of $M$. fructicola, (ii) to investigate the stability of propiconazole sensitivity and fitness parameters in these isolates after consecutive transfers, and (iii) to determine the stability of propiconazole insensitivity in M. fructicola isolates, following periods of long-term storage.

\section{MATERIALS AND METHODS}

In the experiments described below, the fitness of two M. fructicola populations with differing sensitivity to propiconazole was evaluated. Fitness parameters included spore germination, spore production, infection period, and latent period. The impact of subculturing on fungicide sensitivity and selected fitness parameters as well as the effect of cold storage of $M$. fructicola isolates on propiconazole sensitivity were investigated.

Collection and preparation of single-spore isolates. In June and July 2004, M. fructicola isolations were made from brown rot-infected peaches collected from two orchards in Peach County, GA (resistant isolates) and an orchard in Edgefield County, SC (sensitive isolates). Only the Georgia isolates had a history of brown rot control failure with the use of DMI fungicide applications (24); this collection of isolates was confirmed to be resistant to propiconazole later in this study. Conidia from 12 diseased fruit at each orchard were swabbed with individually wrapped sterile cotton tipped applicators (Puritan Medical Products, Guilford, ME), returned to the sterile wrapping, and shipped overnight to Clemson University (Clemson, SC). Upon arrival, applicators were stored dry at $5^{\circ} \mathrm{C}$, until single-spore isolation in October 2004. Single-spore isolation was accomplished by first swirling the tip of each applicator in $1 \mathrm{ml}$ of sterile water containing $0.5 \%$ Tween 20 to create a conidial suspension. Suspensions were vortexed, and $30 \mu \mathrm{l}$ of each suspension was spread on a $15 \times 90 \mathrm{~mm}$ petri dish containing $1.5 \%$ water agar amended with $0.1 \mathrm{mg} / \mathrm{ml}$ streptomycin solution. Hyphal tips from germinating conidia were transferred aseptically to potato dextrose agar (PDA; EMD Chemicals, Gibbstown, NJ). One single-spore isolate was made for each fruit swabbed at the orchard site. Stock cultures of each isolate were prepared on PDA slants immediately after isolation and stored at $5^{\circ} \mathrm{C}$ under sterile light mineral oil prior to formal experimentation.

In vitro sensitivity of isolates. The single-spore isolates of $M$. fructicola were assayed for in vitro sensitivity to propiconazole at the time of isolation and several times thereafter to investigate the influence of consecutive transferring from media to media on fungicide sensitivity. In all experiments, three agar plugs ( $5 \mathrm{~mm}$ in diameter) taken from the periphery of 3-dayold cultures were placed on PDA plates $(15 \times 90 \mathrm{~mm})$ amended with $0.3 \mathrm{mg} / \mathrm{liter}$ propiconazole (Orbit $3.6 \mathrm{EC}$; Syngenta, Greensboro, NC). The concentration of $0.3 \mathrm{mg}$ of propiconazole per liter of medium was used as a discriminatory dose for propiconazole sensitivity testing based on the baseline sensitivity for M. fructicola in South Carolina (30). Two fungicide-amended and two control plates were prepared for each isolate. Colony diameters were measured after 2 days of incubation in the dark at $25^{\circ} \mathrm{C}$. Percent inhibition by propiconazole was calculated by expressing the growth of isolates on amended media as a percentage of the growth on nonamended media and subtracting from $100 \%$. The growth rate of isolates on nonamended media was determined by measuring the change in colony diameter over 3 days. Percent inhibition and growth rate values for each collection of isolates were calculated as the mean of six colony observations from each isolate across four and three experimental replications, respectively.

Comparison of sporulation and conidial germination of isolates. Sporulation and conidial germination frequency were evaluated as components of $M$. fructicola fitness. Yellow cling stone peach halves, no sugar added, in pear juice (Great Value brand, Wal-Mart Stores Inc., Bentonville, AR) were used as the sporulation media. Peach halves were placed cut side down in $6 \times$ $6 \times 10 \mathrm{~cm}$ sterile Magenta boxes (Magenta, Chicago, IL) with one peach half per box. The outer surfaces of four peach halves were inoculated with one $5 \mathrm{~mm}$ agar plug taken from the margins of actively grown colonies on PDA used in the in vitro propiconazole sensitivity experiments. Lesion diameters were measured and spores were collected after 5 days of growth at room temperature $\left(25^{\circ} \mathrm{C}\right)$ under ambient light. Four circular areas ( $5 \mathrm{~mm}$ in diameter) were designated in the centers of the most densely sporulating areas of the peach surface using a $5 \mathrm{~mm}$ sterile cork borer. Spores were scraped from these areas and suspended separately in 1-ml aliquots of sterile water containing $0.5 \%$ Tween 20. Spore concentrations were determined by counting the spores in each suspension using a hemacytometer. Mean spore concentration values for each collection of isolates were calculated based on counts of four sporulating lesion samples from 12 isolates with four experimental replications. At the same time, spore concentration was determined, $20 \mu \mathrm{l}$ of each spore suspension was spread on $1.5 \%$ water agar and incubated at $25^{\circ} \mathrm{C}$ in the dark until spores began to germinate. The frequency of germination for each collection of isolates was calculated by evaluating 100 arbitrarily-selected spores from four spore suspensions for each isolate with four experimental replications.

Comparison of incubation and latent periods of isolates. The incubation and latent periods of propiconazole-sensitive and -resistant isolates of $M$. fructicola were evaluated on detached peach fruit as additional fitness components. The 24 isolates from Georgia and South Carolina were transferred from stock cultures to PDA plates in order to obtain actively growing cultures. Inoculum plates were then prepared by transferring agar plugs from these cultures to fresh PDA plates. To induce conidia production for fruit inoculation, inoculum plates were grown for 3 days at $21^{\circ} \mathrm{C}$ and then transferred onto peach halves as described above.

To prepare fruit for inoculation, yellow cling-stone peaches imported from Chile (Wal-Mart Stores Inc.) were gently hand cleaned with Kimwipes EX-L (Kimberley-Clark Inc., Mississauga, ON) moistened with $70 \%$ ethanol to facilitate removal of chemical residues and trichomes remaining after packing, and to partially sanitize the fruit for inoculation. Fruit were placed in a 1202 bedding plant cell insert (Superior Growers Supply Inc., East Lansing, MI) and set into a 1020 no-holes propagation flat (Superior Growers Supply Inc.) containing paper towels moistened with $300 \mathrm{ml}$ of sterile water. Fruit were stabinoculated at three equidistant points to a depth of $9.5 \mathrm{~mm}$ using a $26 \mathrm{G}^{3 / 8} 9.5 \mathrm{~mm}$ beveled syringe tip (Becton Dickson \& Co., Rutherford, NJ). A $20 \mu \mathrm{l}$ drop of a $4 \times 10^{5}$ conidia $/ \mathrm{ml}$ suspension of M. fructicola was placed at each of the wounds. Once inoculated, Permanest propagation humidity domes (Superior Growers Supply Inc.) were fitted over the propagation flats and sealed with transparent packing tape to promote high humidity.

The fruit were monitored every $3 \mathrm{~h}$ for the appearance of brown rot symptoms and signs (i.e., mycelium and sporulation). The incubation period (i.e., the time from infection to the initial appearance of symptoms [3]), and the latent period (i.e., the time from infection to the first signs of reproductive activity [3]), were determined for each of the inoculated fruit. Mean incubation and latent period values for each collection of isolates were calculated based on observations of three fruit from 12 isolates with four experimental replications.

Impact of consecutive transfers on fitness of isolates. The impact of consecutive transfers on fungicide sensitivity, growth rate, spore germination, and spore production of $M$. fructicola was determined to investigate the legitimacy of maintaining active cultures for experimentation purposes. The aforementioned fitness parameters were evaluated a total of five times with each evaluation occurring after a designated number of consecutive 

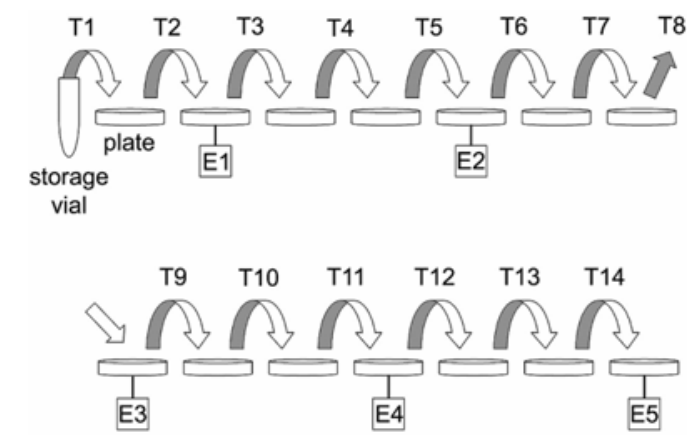

Fig. 1. Schematic of consecutive transfers used for Monilinia fructicola isolates from cold storage to the last evaluation period (E5). Displayed are consecutive transfer events (T1-T14) and the timing of the five evaluations (E1-E5).

transfer events (Fig. 1). Isolates were initially transferred from stock cultures in storage vials to PDA plates in order to obtain actively growing cultures. Agar plugs were transferred from these cultures to fresh PDA plates, which were grown for 3 days at $25^{\circ} \mathrm{C}$ to be used as the starting material for the first evaluation (Fig. 1E1). Between each of the four subsequent evaluations (Fig. 1E2-E5), three successive transfers were made at 7-day intervals. There was a total of 14 transfers over 87 days between the revival of the stock culture and the fifth evaluation. During each evaluation, mean estimates of fitness parameters (fungicide sensitivity, growth rate, spore germination, and spore production) were determined for each collection of isolates as described in the above experiments.

Cold storage effects on propiconazole-resistant isolates. The effects of cold storage on propiconazole sensitivity in $M$. fructicola was investigated. Isolates used in this experiment were collected in 2003 from orchards in Macon and Peach Counties, GA, as part of a previous study (24). Ten isolates were selected based on their insensitivity to propiconazole, the availability of sensitivity data for previous years, and their time in storage. Isolates were assayed for propiconazole sensitivity at the time of isolation, and at 8 and 34 months after storage. All isolates had been stored on PDA slants at $5^{\circ} \mathrm{C}$ under sterile light mineral oil, and were initially transferred from stock cultures to PDA plates in order to obtain actively growing cultures prior to experimentation. Propiconazole sensitivity was determined by percent inhibition on media amended with $0.3 \mathrm{mg}$ of propiconazole per liter of medium as described in the procedures above. Inhibition values were expressed as the mean of six colony observations of the 10 isolates with four replications.

Data Analysis. Data from the fitness, incubation period, and latent period experiments were subjected to analysis of variance (ANOVA) for a randomized block design using the General Linear Model (GLM) procedure of SAS (ver. 9.1; SAS Institute Inc., Cary, NC); all experimental replications were considered blocks in the ANOVA. The consecutive transfer experimental data were also analyzed by ANOVA for a factorial design using SAS (SAS Institute Inc.) with isolate sensitivity (resistant or sensitive) and number of consecutive transfer events as the two treatment factors. All analyses of percentage data were based on arcsinesquare root-transformed percentage values. Main effects and interactions in analyses were considered statistically significant at $\alpha=0.05$.

\section{RESULTS}

Initial propiconazole sensitivity of single-spore isolates of M. fructicola from Georgia and South Carolina. Sensitivity to propiconazole at the initial evaluation (Fig. 1 E1) was different for the South Carolina and Georgia isolates. South Carolina isolates did not grow at the discriminatory dose of $0.3 \mathrm{mg} / \mathrm{liter}$

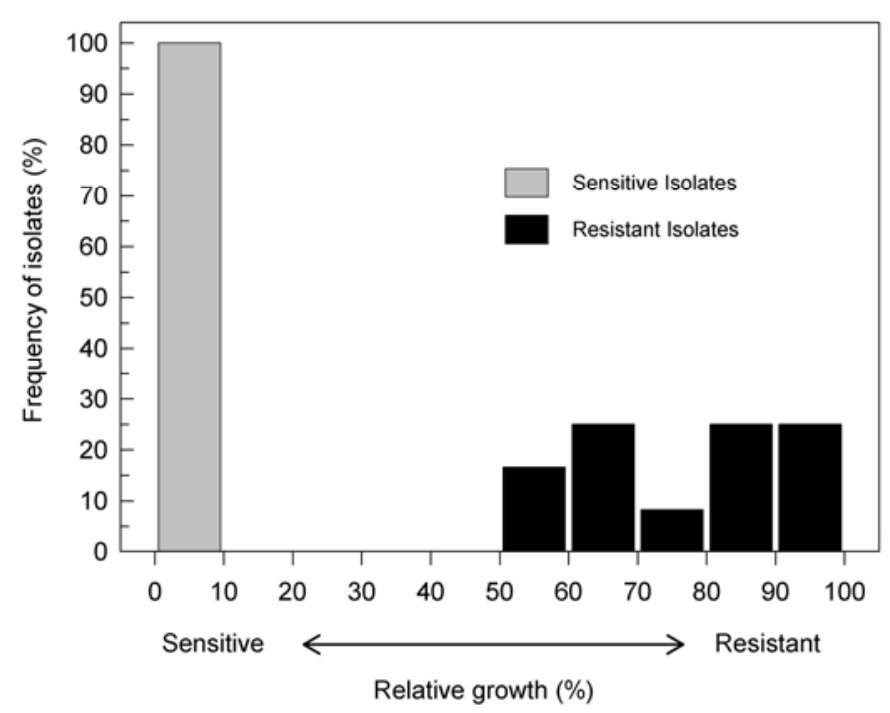

Fig. 2. Propiconazole sensitivity frequency distribution of sensitive and resistant Monilinia fructicola isolates at the time of collection. Sensitivity is expressed as radial mycelial growth on propiconazole-amended medium $(0.3$ $\mathrm{mg} / \mathrm{liter}$ ) relative to growth on unamended medium. Values are means of 12 isolates with three replications.

propiconazole, and hence, the isolate collection was designated sensitive (Fig. 2). The Georgia isolates were more widely distributed in regard to propiconazole sensitivity (Fig. 2). At the $0.3 \mathrm{mg} /$ liter discriminatory dose, the Georgia isolates fell between 57.6 and $97.4 \%$ radial growth relative to the control. Since Georgia isolates exhibited substantial radial growth at the discriminatory dose, the isolate collection was designated resistant (Fig. 2).

Initial fitness of propiconazole-resistant and -sensitive isolates. At the first evaluation (Fig. $1 \mathrm{E} 1$ ), all isolates from both collections of isolates sporulated on peach halves and produced viable conidia. Across both isolate collections, on average, conidia germinated with a frequency greater than $84 \%$ and were produced in concentrations in excess of $3.49 \times 10^{5}$ conidia/ml (Table 1). There were no differences in the frequency of germination $(P=0.214)$ and the amount of sporulation $(P=$ 0.558 ) between propiconazole-resistant and -sensitive isolates.

The latent and incubation periods were also similar between the resistant and sensitive isolates. All isolates from both collections produced symptoms and became reproductive on detached peach fruit within $60 \mathrm{~h}$ after inoculation. For the sensitive isolates, it took on average $31.41 \mathrm{~h}$ from the time of inoculation to the first appearance of brown rot lesions, and $54.34 \mathrm{~h}$ until lesions became reproductive. By comparison, the resistant isolates had an average incubation and latent period of 33.94 and $53.11 \mathrm{~h}$, respectively. There was a small but significant $(P=0.044)$ difference in incubation period between the resistant and sensitive isolates, with the sensitive isolates having a slightly shorter incubation period than the resistant isolates. Although latent period was shorter on average for resistant isolates, the difference was not significant $(P=0.384)$.

The influence of consecutive transfers on the fitness of propiconazole-sensitive and -resistant isolates. The fitness of propiconazole-sensitive and -resistant $M$. fructicola isolates was influenced by consecutive transfers. When analyzed across all transfers, the sensitive isolates had higher spore germination $(P=$ $0.025)$ and spore production $(P=0.025)$ compared to the resistant isolates (Table 1). Although the sensitive isolates were more fit in terms of spore germination and spore production, there were no differences in growth rate between the two collections of isolates $(P=0.424)$. There was considerable variability for all three fitness parameters (spore production, germination, and growth rate) 


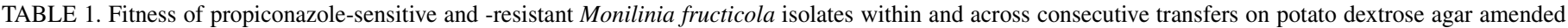
with $0.3 \mathrm{mg}$ of propiconazole per liter of medium

\begin{tabular}{|c|c|c|c|c|c|}
\hline \multirow[b]{2}{*}{ Evaluation $^{v}$} & \multirow[b]{2}{*}{ Isolate collection } & \multirow[b]{2}{*}{ No. of isolates } & \multicolumn{3}{|c|}{ Fitness parameters $^{\mathrm{w}}$} \\
\hline & & & Spore germination $(\%)$ & $\begin{array}{c}\text { Spore production } \\
\text { (spores per milliliter) }\end{array}$ & $\begin{array}{c}\text { Growth rate } \\
(\text { millimeters per hour })^{\mathrm{x}}\end{array}$ \\
\hline $\begin{array}{l}\text { E1 } \\
\text { (Initial) }\end{array}$ & $\begin{array}{l}\text { Sensitive } \\
\text { Resistant }\end{array}$ & $\begin{array}{l}12 \\
12\end{array}$ & $\begin{array}{l}91.95 \pm 3.3 \mathrm{a} \\
84.62 \pm 5.0 \mathrm{a}\end{array}$ & $\begin{array}{l}4.24 \times 10^{5} \pm 1.0 \times 10^{5} \mathrm{a} \\
3.49 \times 10^{5} \pm 0.8 \times 10^{5} \mathrm{a}\end{array}$ & $\begin{array}{l}--y \\
--y\end{array}$ \\
\hline E2 & $\begin{array}{l}\text { Sensitive } \\
\text { Resistant }\end{array}$ & $\begin{array}{l}12 \\
12\end{array}$ & $\begin{array}{l}96.30 \pm 0.8 \mathrm{a} \\
88.07 \pm 3.7 \mathrm{~b}\end{array}$ & $\begin{array}{l}33.33 \times 10^{5} \pm 4.0 \times 10^{5} \mathrm{a} \\
25.28 \times 10^{5} \pm 2.6 \times 10^{5} \mathrm{a}\end{array}$ & $\begin{array}{l}0.86 \pm 0.04 \mathrm{a} \\
0.89 \pm 0.05 \mathrm{a}\end{array}$ \\
\hline E3 & $\begin{array}{l}\text { Sensitive } \\
\text { Resistant }\end{array}$ & $\begin{array}{l}12 \\
12\end{array}$ & $\begin{array}{l}82.08 \pm 4.5 \mathrm{a} \\
85.20 \pm 6.3 \mathrm{a}\end{array}$ & $\begin{array}{l}9.42 \times 10^{5} \pm 3.7 \times 10^{5} \mathrm{a} \\
1.10 \times 10^{5} \pm 0.3 \times 10^{5} \mathrm{~b}\end{array}$ & $\begin{array}{l}0.38 \pm 0.03 \mathrm{a} \\
0.42 \pm 0.03 \mathrm{a}\end{array}$ \\
\hline $\mathrm{E} 4$ & $\begin{array}{l}\text { Sensitive } \\
\text { Resistant }\end{array}$ & $\begin{array}{l}12 \\
12\end{array}$ & $\begin{array}{l}83.86 \pm 6.1 \mathrm{a} \\
80.96 \pm 5.1 \mathrm{a}\end{array}$ & $\begin{array}{l}4.76 \times 10^{5} \pm 1.4 \times 10^{5} \mathrm{a} \\
4.08 \times 10^{5} \pm 1.2 \times 10^{5} \mathrm{a}\end{array}$ & $\begin{array}{l}0.42 \pm 0.02 \mathrm{a} \\
0.43 \pm 0.03 \mathrm{a}\end{array}$ \\
\hline E5 & $\begin{array}{l}\text { Sensitive } \\
\text { Resistant }\end{array}$ & $\begin{array}{l}12 \\
12\end{array}$ & $\begin{array}{l}59.83 \pm 9.3 \mathrm{a} \\
52.81 \pm 6.4 \mathrm{a}\end{array}$ & $\begin{array}{l}1.20 \times 10^{5} \pm 0.4 \times 10^{5} \mathrm{a} \\
1.92 \times 10^{5} \pm 0.5 \times 10^{5} \mathrm{a}\end{array}$ & $\begin{array}{l}0.55 \pm 0.02 \mathrm{a} \\
0.53 \pm 0.03 \mathrm{a}\end{array}$ \\
\hline $\mathrm{All}^{\mathrm{z}}$ & $\begin{array}{l}\text { Sensitive } \\
\text { Resistant }\end{array}$ & $\begin{array}{l}12 \\
12\end{array}$ & $\begin{array}{l}88.52 \pm 1.9 \mathrm{a} \\
79.62 \pm 2.5 \mathrm{~b}\end{array}$ & $\begin{array}{r}10.57 \times 10^{5} \pm 1.4 \times 10^{5} \mathrm{a} \\
7.17 \times 10^{5} \pm 0.9 \times 10^{5} \mathrm{~b}\end{array}$ & $\begin{array}{l}0.55 \pm 0.11 \mathrm{a} \\
0.57 \pm 0.11 \mathrm{a}\end{array}$ \\
\hline
\end{tabular}

${ }^{\mathrm{v}}$ Indicates five evaluations of the fitness parameters for the two isolate collections.

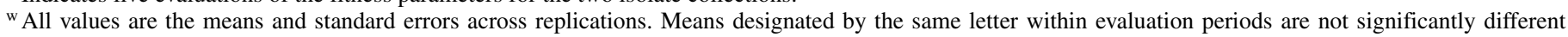
according to Fisher's protected least significant difference test $(\alpha=0.05)$.

${ }^{x}$ Growth rate (millimeters per hour) is expressed as mean population growth.

y No data were available for the first evaluation period.

${ }^{z}$ Indicates values averaged and analyzed across all transfers.

among the five evaluation periods (Fig. 1), which occurred after 3, $5,8,11$, and 14 consecutive transfers, respectively (Table 1). There was an overall decrease in the three fitness parameters for isolates from both collections over consecutive transfers, but this decrease was not consistent across evaluation periods (Table 1). When analyzed as a factor in the experimental design, the number of consecutive transfers was found to be significant for spore production, germination, and growth rate $(P<0.0001)$. Comparisons within evaluation periods indicated that there were no significant differences between sensitive and resistant isolates for the fitness parameters (Table 1). Spore germination in the second evaluation $(P=0.030)$ and spore production in the third evaluation $(P=$ $0.034)$ were the only exceptions as they were significantly higher in the sensitive isolates compared with the resistant isolates (Table 1).

In regard to propiconazole sensitivity, the number of consecutive transfers and the interaction between propiconazole sensitivity and the number of consecutive transfers were both significant $(P<0.0001)$. For the sensitive isolates, sensitivity to propiconazole remained consistent over consecutive transfers (Fig. 3). In contrast, percent inhibition at $0.3 \mathrm{mg} / \mathrm{liter}$ propiconazole initially increased in the resistant isolates, but leveled off with consecutive transfers (Fig. 3). Despite the loss in resistance over consecutive transfers, the resistant isolates remained more resistant to propiconazole (i.e., had lower percent inhibition) than the sensitive isolates $(P<0.0001)$ (Fig. 3).

Effects of cold storage on propiconazole-resistant isolates. When initially tested in 2003, the sensitivity of the 10 isolates collected from Macon and Peach Counties, Georgia, at the discriminatory dose of $0.3 \mathrm{mg} /$ liter of propiconazole ranged from 0.83 to $34.44 \%$ inhibition of radial growth compared to the control (unamended media). When tested after several months of cold storage on PDA slants, the sensitivity of the isolates had increased (Fig. 4). Percent inhibition had increased by $165 \%$ and $273 \%$ after 8 and 34 months of cold storage, respectively.

\section{DISCUSSION}

The M. fructicola isolates from Georgia and South Carolina differed in propiconazole sensitivity, but were not different in terms of vegetative and reproductive fitness or ability to cause disease with the exception of incubation period. While the level of resistance to propiconazole varied among the Georgia isolates, the isolates from South Carolina exhibited little variation in sen-

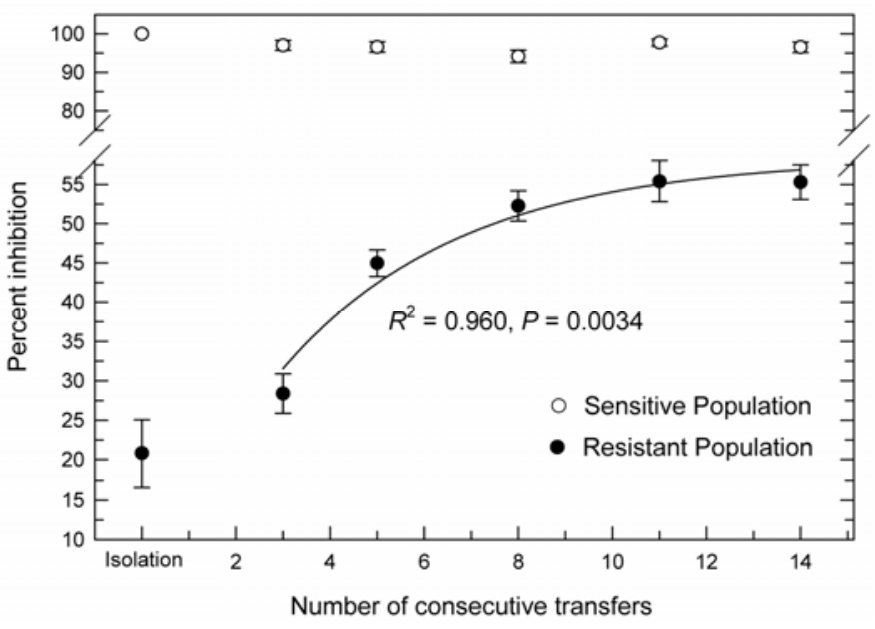

Fig. 3. Propiconazole sensitivity over consecutive transfers for sensitive and resistant isolates of Monilinia fructicola. Propiconazole sensitivity is expressed as percent inhibition of mycelial growth on potato dextrose agar (PDA) amended with $0.3 \mathrm{mg} /$ liter propiconazole relative to growth on unamended PDA. Values are the means and standard errors of six colony observations from 12 isolates with four replications. Points at the time designated "Isolation" represent the means and standard errors of propiconazole sensitivity at the time of isolation prior to storage and the first transfer event (T1) (Fig. 1). An exponential rise to maximum distribution function $\left[y=\mathrm{a}\left(1-e^{-\mathrm{bx}}\right)\right]$ was fitted to means of percent inhibition for the resistant population over the course of the transfer experiment. Adjusted $R^{2}$ value was $0.960(n=5 ; P=0.0034)$.

sitivity to propiconazole. The wide range of sensitivity to propiconazole in the Georgia isolates may be indicative of the slow shift in sensitivity characteristic of DMI resistance development $(12,13,24,30)$. There was some variability in spore production across evaluation periods, but this variability was present across all isolates. The extremely high levels of sporulation observed during the second evaluation (E2) may have been due to a power failure that occurred in the lab for a brief period that coincided with the evaluation. Similar to our results, benzimidazole-tolerant and -sensitive $M$. fructicola isolates were not different regarding sporulation, spore germination, and growth rate in vitro (20). In contrast, dicarboximide-resistant isolates of $M$. fructicola were shown to have reduced sporulation on detached fruit (22). In another ascomycete, DMI-resistant isolates of Cercospora beticola were similar to DMI-sensitive isolates in 
regard to spore germination, germ tube length, and mycelial growth, but had reduced sporulation (11). Propiconazole-adapted isolates of the ascomycete Pyrenophora tritici-repentis were shown to have a reduced growth rate on agar medium (21). Clearly, vegetative and reproductive fitness costs associated with fungicide resistance in ascomycetes are highly specific to the fungal species, fitness parameters, and the fungicide in question, as studies indicate no consistent trends with regard to fungicide resistance and fitness.

The incubation period of the sensitive isolates was slightly shorter than that of resistant isolates, but there were no other differences in ability to cause disease. Similarly, DMI-resistant isolates of Rhynchosporium secalis were found to be no less pathogenic on barley leaves than DMI-sensitive isolates (12). In contrast, DMI-resistant isolates of $C$. beticola were reported to have a similar incubation period, but reduced virulence, on sugar beet compared to DMI-sensitive isolates (11). In M. fructicola, dicarboximide-resistant isolates were reported to produce smaller lesions and sporulate less on peach fruit (22). By comparison, benzimidazole-resistant isolates of $M$. fructicola were shown to be equally pathogenic and competitive with sensitive isolates on peach fruit. As a consequence, benzimidazole resistance has been persistent in South Carolina despite infrequent applications of benzimidazoles (31). By itself, uncompromised pathogenicity in propiconazole-resistant isolates may be an indication that propiconazole resistance could exist stably in populations of M. fructicola. However, as part of this study we found that resistance is being lost in vitro in the absence of DMI pressure.

Propiconazole resistance decreased considerably with consecutive transferring and after cold storage. Similar losses in fungicide resistance following consecutive transferring has been reported for several fungal pathosystems $(4,10,11,15,19)$. For example, QoI-resistant isolates of Plasmopara viticola were found to revert to complete sensitivity after consecutive transfers on untreated grapes (4), and following subculturing on wheat, only 10 of 46 QoI-resistant isolates of Mycosphaerella graminicola were reported to retain fungicide resistance (19). With DMI fungicides, flutriafol resistance in C. beticola isolates remained stable for only 10 consecutive transfers over 5 months on agar media and on sugar beets in Greece (10). Similarly, flusilazole resistance in Venturia inaequalis was found to be stable over 6 months of consecutive transferring at $20^{\circ} \mathrm{C}$, but became unstable

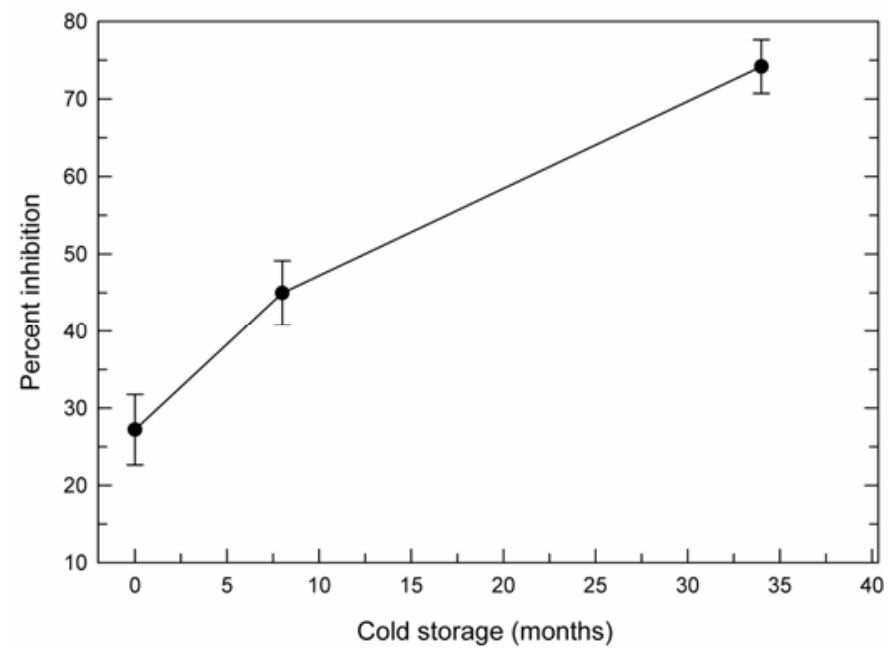

Fig. 4. Increase in propiconazole sensitivity after periods of cold storage on potato dextrose agar (PDA) slants for propiconazole-resistant isolates of Monilinia fructicola. Propiconazole sensitivity is expressed as percent inhibition of fungal growth on PDA plates amended with $0.3 \mathrm{mg} / \mathrm{liter}$ propiconazole relative to growth on unamended PDA. Values are the means and standard errors of two colony observations from 10 isolates with three replications. after 10 months of consecutive transferring, with resistant isolates displaying the highest degree of instability (14). By comparison, the sensitivity of propiconazole-resistant $M$. fructicola isolates used in the present study began to increase as soon as 10 days after consecutive transferring. Perhaps flutriafol and flusilazole resistance are more stable traits in Greek isolates of C. beticola and New York State isolates of $V$. inaequalis, respectively, compared with propiconazole resistance in M. fructicola isolates in the southeastern United States. In regard to cold storage, flutriafol resistance in $C$. beticola isolates decreased by factors ranging from one to fivefold after 10 months in storage. A decrease in flusilazole resistance following 7 months of cold storage at $2^{\circ} \mathrm{C}$ was also reported for isolates of Venturia inaequalis (14). We observed a similar decline in resistance in propiconazole-resistant $M$. fructicola isolates after 8 months under similar cold storage conditions. Moreover, after longer periods of cold storage (34 months), the decline in resistance of resistant isolates was even more pronounced. A high degree of instability exists for DMI resistance in $V$. inaequalis and C. beticola, and environmental factors such as cold may exacerbate this instability. This instability also appears to be present for DMI resistance in $M$. fructicola. However, the instability and environmental impacts are more extreme in $M$. fructicola compared with that in $V$. inaequalis (14), as sensitivity changes in resistant isolates were equally drastic within a shorter time frame.

Instability in propiconazole resistance may have important implications for DMI resistance management of $M$. fructicola in the southeastern United Sates. Although similar fitness between sensitive and resistant isolates points toward long term stability of DMI resistance in the field, the instability of resistance in the absence of DMI pressure would indicate an opposite trend. The instability may not result in a complete reversion to the base line sensitivity, but may allow for resistant populations to shift towards increased sensitivity under management programs with reduced DMI inputs. At the same time, the lack of fitness costs associated with propiconazole resistance observed in this study could balance the observed instability of resistance, allowing for $M$. fructicola populations to maintain a moderate level of resistance even under programs with no or reduced DMI input.

The rapid increase in sensitivity in propiconazole-resistant M. fructicola isolates following a short number of consecutive transfers and short periods in cold storage may provide some additional insight into the mechanism of DMI resistance in this system. These increases in the propiconazole sensitivity occurred too quickly to be attributed to mutations in the cytochrome P450 sterol $14 \alpha$-demythylase gene (CYP51), especially in the absence of a mutagen. If mutations in the CYP51 gene were the mechanism of resistance in this instance, one would have expected a more qualitative shift in the sensitivity of resistant isolates with a complete reversion to the base line sensitivity. In support of our hypothesis, several of the isolates from the resistant $M$. fructicola population used in the current study had only a few nucleotide mutations in the coding region of the CYP51 gene, but none of the mutations resulted in a corresponding amino acid change (17). This may be an indication that other documented mechanisms of DMI resistance may be involved, such as overexpression of the CYP51 gene $(6,16,25,26)$ or overexpression of ATP-binding cassettes encoding efflux pumps $(7,32)$.

In short, we found that propiconazole-resistant isolates were not compromised in vegetative and reproductive fitness and ability to cause disease compared with -sensitive isolates at the time of collection. However, fitness decreased in resistant isolation over consecutive transfers and propiconazole resistance became unstable. The instability of propiconazole resistance in M. fructicola may have important implications for fungicide 
resistance management, in that sensitivity is likely to return in resistant populations in the absence of DMI pressure.

\section{ACKNOWLEDGMENTS}

We thank P. Brannen, Univ. Georgia, Athens, for providing isolates from Georgia. This study was supported in part by the Cooperative State Research, Education, and Extension Service (CSREES) of the United States Department of Agriculture (USDA) and a grant from the USDACSREES Risk Avoidance and Mitigation (RAMP) program. This is technical contribution no. 5229 of the Clemson University Experiment Station, Clemson, SC.

\section{LITERATURE CITED}

1. Batra, L. R. 1991. World species of Monilinia (fungi): Their ecology, biosystematics, and control. New York Botanical Garden \& Mycological Society of America, New York. pp. 66-80.

2. Brannen, P. M., Hotchkiss, M., Reilly, C. C., and Schnabel, G. 2005. Evaluation of fungicide programs to manage a DMI-resistant Monilinia fructicola population in a Georgia peach research block, 2005. F\&N Tests 61:STF001

3. Campbell, C. L., and Madden, L. V. 1990. Disease or Epidemic Components. Pages 216-217 in: Introduction to Plant Disease Epidemiology, John Wiley \& Sons, New York.

4. Genet, J.-L., Jaworska, G., and Deparis, F. 2006. Effect of dose rate and mixtures of fungicides on selection for QoI resistance in populations of Plasmopara viticola. Pest Manag. Sci. 62:188-194.

5. Gullino, M. L., and Garibaldi, A. 1979. Experimental observations on resistance to vinclozolin in Italian isolates of Botrytis cinerea. Dif. Piante 2:341-350 (Abstr.) Rev. Plant Pathol. 360:579.

6. Hamamoto, H., Hasegawa, K., Nakaune, R., Lee, Y. J., Makizumi, Y., Akutsu, K., and Hibi, T. 2000. Tandem repeat of a transcriptional enhancer upstream of the sterol $14 \alpha$-demethylase gene (CYP51) in Penicillium digitatum. App. Environ. Microbiol. 66:3421-3426.

7. Hayashi, K., Schoonbeck, H., and De Waard, M. A. 2006. Expression of the $\mathrm{ABC}$ transporter BcatrD from Botrytis cinerea reduces sensitivity to sterol demethylation inhibitor fungicides. Pestic. Biochem. Physiol. 73:110-121.

8. Horton, D., Brannen, P., Bellinger, B., and Ritchie, D. 2006. Southern peach, nectarine, and plum pest management and culture guide. D. Horton, P. Brannen, B. Bellinger, and D. Ritchie, eds. University of Georgia, Athens. pp. 6-18.

9. Jones, A. L., and Ehret, G. R. 1976. Isolation and characterization of benomyltolerant strains of Monilinia fructicola. Plant Dis. Rep. 60:765-769.

10. Karaoglanidis, G. S., and Thanassoulopoulos, C. C. 2002. Phenotypic instability of Cercospora beticola sacc. strains expressing resistance to the sterol demethylation-inhibiting (DMI) fungicide flutriafol after cold exposure. J. Phytopathol. 150:692-696.

11. Karaoglanidis, G. S., Thanassoulopoulos, C. C., and Ioannidis, P. M. 2001. Fitness of Cercospora beticola field isolates resistant and sensitive to demethylation inhibitor fungicides. Eur. J. Plant Pathol. 107:337-347.

12. Kendall, S. J., Hollomon, D. W., Cooke, L. R., and Jonse, D. R. 1993. Changes in sensitivity to DMI fungicides in Rhynchosporium secalis. Crop Prot. 12:357-362.

13. Köller, W. 2001. Fungicide resistance. Pages 483-488 in: Encyclopedia of Plant Pathology, O. C. Maloy and T. D. Murray, eds. John Wiley \& Sons, NY.

14. Köller, W., Smith, F. D., and Reynolds, K. L. 1991. Phenotypic instability of flusilazole sensitivity in Venturia inaequalis. Plant Pathol. 40:608-611.
15. Lim, T. H., Pryor, B. M., Morgan, D. P., and Michailides, T. J. 2003. Sensitivity to iprodione, vinclozolin, and tebuconazole and characters of iprodione-resistant isolates of Alternaria spp. from pistachio. (Abstr.) Phytopathology 93(Suppl.):S128.

16. Ma, Z., Proffer, T. J., Jacobs, J. L., and Sundin, W. 2006. Overexpression of the 14a-demethylase target gene (CYP51) mediates fungicide resistance in Blumeriella jaapii. Appl. Environ. Microbiol. 72:2581-2585.

17. McClive, C., and Schnabel, G. 2005. Evaluation of the CYP51 gene from Monilinia fructicola as a genetic determinant of DMI fungicide resistance. (Poster). Clemson University Undergraduate Research Conference, Clemson University, Clemson, SC.

18. McGee, D. C., and Zuck, M. G. 1981. Competition between benomylresistant and -sensitive strains of Venturia inaequalis on apple seedlings. Phytopathology 70:8-12.

19. Miguez, M., Reeve, C., Wood, P. M., and Hollomon, D. W. 2004. Alternative oxidase reduces the sensitivity of Mycosphaerella graminicola to QoI fungicides. Pest Manag. Sci. 60:3-7.

20. Penrose, L. J., Davis, K. C., and Koffmann, W. 1979. The distribution of benomyl-tolerant Sclerotinia fructicola (Wint.) Rhem. in stone fruit orchards in New South Wales and comparative studies with susceptible isolates. Aust. J. Agric. Res. 30:307-319.

21. Reimann, S., and Deising, H. B. 2005. Inhibition of efflux transportermediated fungicide resistance in Pyrenophora tritici-repentis by a derivative of $4^{\prime}$-hydroxyflavone and enhancement of fungicide activity. Appl. Environ. Microbiol. 71:3269-3275.

22. Ritchie, D. 1983. Mycelial growth, peach fruit rotting capability, and sporulation of strains of Monilinia fructicola resistant to Dichloran, Iprodione, Procymidone, and Vinclozolin. Phytopathology 73:44-47.

23. Rosenberger, D. A. 1981. Postharvest fungicides for apples: Development of resistance to Benomyl, Vinclozolin, and Iprodione. Plant Dis. 65:10101013.

24. Schnabel, G., Bryson, P. K., Bridges, W. C., and Brannen, P. M. 2004. Reduced sensitivity in Monilinia fructicola to propiconazole in Georgia and implications for disease management. Plant Dis. 88:1000-1004.

25. Schnabel, G., and Dai, Q. 2004. Heterologous expression of the P450 sterol $14 \alpha$-demethylase gene from Monilinia fructicola reduces sensitivity to some but not all DMI fungicides. Pest. Biochem. Phys. 78:3138 .

26. Schnabel, G., and Jones, A. L. 2001. The 14 $\alpha$-Demethylase (CYP51A1) Gene is Overexpressed in Venturia inaequalis Strains Resistant to Myclobutanil. Phytopathology 91:102-110.

27. Schuepp, H., and Kueng, M. 1981. Stability and tolerance to MBC in populations of Botrytis cinerea in vineyards of northern and eastern Switzerland. Can. J. Plant Pathol. 3:180-181.

28. Takahashi, Y., Ichihashi, Y., Sano, T., and Harada, Y. 2004. Monilinia jezoensis sp. nov. in the Sclerotiniaceae, causing leaf blight and mummy fruit disease of Rhododendron kaempferi in Hokkaido, northern Japan. Mycoscience. 46:106-109.

29. Webster, R. K., Ogawa, J. M., and Bose, E. 1970. Tolerance of Botrytis cinerea to 2,6-dichloro-4-nitroaniline. Phytopathology 60:1489-1492.

30. Zehr, E., Luszcz, L. A., Olien, W. C., Newall, W. C., and Toler, J. E. 1999. Reduced sensitivity in Monilinia fructicola to propiconazole following prolonged exposure in peach orchards. Plant Dis. 83:913-916.

31. Zehr, E. I., Toler, J. E., and Luszcz, L. A. 1991. Spread and persistence of benomyl-resistant Monilinia fructicola in South Carolina peach orchards. Plant Dis. 75:590-593.

32. Zwiers, L. H., Sterigopoulos, I., Van Nistelrooy, J. G. M., and De Waard, M. A. 2002. ABC transporters and azole susceptibility on laboratory strains of the wheat pathogen Mycosphaerella graminicola. Antimicrob. Agents Chemother. 46:3900-3906. 